\title{
Identification of novel cancer fusion genes using chromosome breakpoint screening
}

\author{
KATE HUA ${ }^{1,2}$, CHIN-HUI LIN ${ }^{1}$, YA-LUN CHEN ${ }^{1}$, CHI-HUNG LIN ${ }^{1,3}$, YUEH-HSIN PING ${ }^{2}$, \\ YUH-SHAN JOU ${ }^{4}$ and CHIAN-FENG CHEN ${ }^{1}$ \\ ${ }^{1}$ VYM Genome Research Center, ${ }^{2}$ Department and Institute of Pharmacology and \\ ${ }^{3}$ Institute of Microbiology and Immunology, National Yang-Ming University, Taipei 112; \\ ${ }^{4}$ Institute of Biomedical Sciences, Academia Sinica, Taipei 115, Taiwan, R.O.C.
}

Received August 8, 2016; Accepted September 22, 2016

DOI: $10.3892 /$ or.2017.5492

\begin{abstract}
Gene fusion due to rearrangement or translocation of chromosomes is a powerful mutational mechanism during tumorigenesis. Several new high-resolution technologies have recently been developed to evaluate large numbers of small aberrations as candidate loci for fusion gene screening. In our previous whole-genome screening study using 500K SNP arrays, we identified more than 700 homozygous deletions (HDs) and amplicons in 23 cancer cell lines. To explore novel fusion genes in cancer, we established stringent criteria for defining HD and amplicon breakpoints. Then genomic PCR and sequencing analyses identified a fusion gene, FNDC3BPRKCI, that resulted from chromosome intra-rearrangement. Western blotting and 3'-RACE analyses revealed that the chimeric transcript was an in-frame fusion between FNDC3B and PRKCI. Finally, cell migration and colony formation assays suggested that FNDC3B-PRKCI is a potential oncogene.
\end{abstract}

\section{Introduction}

Gene fusion commonly occurs through chromosome rearrangement and translocation during tumorigenesis $(1,2)$. They are powerful 'gain of function' mutations in cancers that alter protein expression, remove regulatory domains, force oligomerization, change subcellular localization or fuse to a new domain (3). Moreover, fusion genes are tumor-specific and have

Correspondence to: Professor Chian-Feng Chen, VYM Genome Research Center, National Yang-Ming University, 155, Section 2, 11221 Linong Street, Taipei 112, Taiwan, R.O.C.

E-mail: cfchen@ym.edu.tw

Abbreviations: HD, homozygous deletion; SNP, single nucleotide polymorphism; FNDC3B, fibronectin type III domain containing 3B; PRKCI, protein kinase $\mathrm{C}$ iota; HCC, hepatocellular carcinoma; RACE, rapid amplification of cDNA ends

Key words: chromosome rearrangement, chromosome breakpoints, fusion gene, FNDC3B, PRKCI been used as diagnostic markers and therapeutic targets (3). For example, $\sim 98 \%$ of patients with acute promyelocytic leukemia carry a translocation of chromosomes 15 and 17, which creates the fusion for retinoic acid receptor $\alpha(\operatorname{RAR} \alpha)$ and promyelocytic leukemia (PML) protein. More than $80 \%$ of patients who carry the RAR $\alpha$-PML fusion achieve prolonged remission after treatment with all-trans retinoic acid (4).

Historically, fusion genes have been mainly associated with hematological and mesenchymal malignancies $(2,5)$. Although, over 440 gene fusions have been identified in benign tumors and cancers, only $\sim 15 \%$ are found in epithelial tumors (6). Discovery of fusion genes has traditionally relied on the detection of translocations using cytogenetic techniques. However, the low resolution of cytogenetic techniques and the complex karyotypes of epithelial tumors make it difficult to identify these translocations in epithelial tumors (3). In addition, gene fusion can also be produced by small intra-chromosomal rearrangements such as deletions, amplifications or insertions that cannot be detected using cytogenetic techniques (3). In recent years, several new non-cytogenetic technologies have been developed to resolve this problem. For example, pair-end sequencing of cancer cDNA using new high-throughput sequencing platforms, high-resolution single nucleotide polymorphism (SNP) arrays and array comparative genomic hybridization $(\mathrm{aCGH})$ can detect small copy number aberrations as candidate fusion loci and be used to suggest the potential breakpoints in cancer genomes $(7,8)$.

In our previous whole-genome screening study using 500K SNP arrays, we identified 57 homozygous deletions (HDs) and 653 amplicons in 23 cancer cell lines (9). These aberrations were produced by either inter-chromosome or intra-chromosome rearrangements, and also created many chromosome breakpoints. Gene fusion can occur if two breakpoints are both located in the gene region. In the present study, we applied the results from our genome-wide SNP arrays to screen for chromosome breakpoints and explore novel fusion genes in several cancer cell lines.

\section{Materials and methods}

Cell lines, cell culture and antibodies. Hep3B, HepG2, HuH6, HuH7, SK-Hep-1 and 293T cells were obtained from the 
American Type Culture Collection (ATCC; Manassas, VA, USA) and cultured in Dulbecco's modified Eagle's medium supplemented with $10 \%$ fetal bovine serum, $1 \%$ non-essential amino acids, and $1 \%$ penicillin/streptomycin (Invitrogen, Carlsbad, CA, USA). Primary anti-FNDC3B and anti-PRKCI antibodies were purchased from Sigma-Aldrich (St. Louis, MO, USA) and BD Biosciences (San Jose, CA, USA), respectively. HRP-conjugated secondary antibodies were purchased from Millipore Corporation (Billerica, MA, USA). The rabbit polyclonal antibody against FNDC3B-PRKCI was generated at LTK BioLaboratories (Taoyuan, Taiwan) by injecting a rabbit with a synthetic oligopeptide near the FNDC3B-PRKCI fusion point (LLEWDEEPVMPM; FNDC3B, amino acids 413-418; and PRKCI, amino acids 198-203).

3'-Rapid amplification of cDNA ends (RACE) PCR. RNA was extracted from Hep3B cells using TRIzol reagent (Invitrogen). The 3'-end of the chimeric transcript was identified using a 3'-RACE System for rapid amplification of cDNA ends kit (Invitrogen). RNA was reverse-transcribed into cDNA using the adapter primer (GGCCACGCGTCGACTAGTACTTTTT TTTTTTTTTTTT). Then, the product was PCR-amplified using a FNDC3B-specific primer (TTCCCATGATGTCACCC AAT) and an abridged universal amplification primer (GGCC ACGCGTCGACTAGTAC).

Analysis of copy number alterations and genomic PCR The copy number alterations were detected by Affymetrix GeneChip Human Mapping 500K SNP Arrays (Affymetrix, Santa Clara, CA, USA) and analysis by dChip software as previously described (9). In brief, the copy number $(\mathrm{CN})$ for an SNP probe in cancer cell lines was computed as follows:

$\mathrm{CN}=(\mathrm{SNP}$ signal in Hep3B/mean signal of the reference at SNP $) \times 2$

To search for $3 \mathrm{q} 26.2$ breakpoints, we designed 3 forward primers for $F N D C 3 B$ intron 12 (F1, AGCTGGGAAGTTCAA GGTCAAGGACTTGTATCTGG, F2, TAAGGGCAGGGAA CCCAAACAGACTGTTTATCTCC, and F3, GATCAGGCT GGGCAGTTTGGGCCAATCTAAGT) to pair with the reverse primer (R, ATGGATGACTGATCCATGGGCATCAC TGGT) for PRKCI exon 7. The PCR product was cloned and sequenced.

Cell migration, cell growth and anchorage-independent growth assays. Full-length FNDC3B-PRKCI was obtained by PCR from Hep3B cell cDNA, and the product was ligated into a pCDNA3.0-HA vector (Invitrogen). Cells for the wound-healing assay were seeded at a confluent density and cultured overnight to permit growth into a monolayer. The cultured cells were scratched with a p200 pipette tip, and the wound was allowed to close for $20 \mathrm{~h}$. The average decrease in the area covered by the cells was calculated to evaluate the rate of wound closure. Cell viability was determined by the cell proliferation assay every $48 \mathrm{~h}$ for 6 days using the AlamarBlue reagent (AbD Serotec, Kidlington, UK), and absorbance values at wavelengths of 560 and $590 \mathrm{~nm}$ were evaluated to calculate the growth curves. Approximately 10,000 cells were mixed in $0.25 \%$ top agarose for the soft agar colony formation assay, and plated onto $0.5 \%$ bottom agarose in a

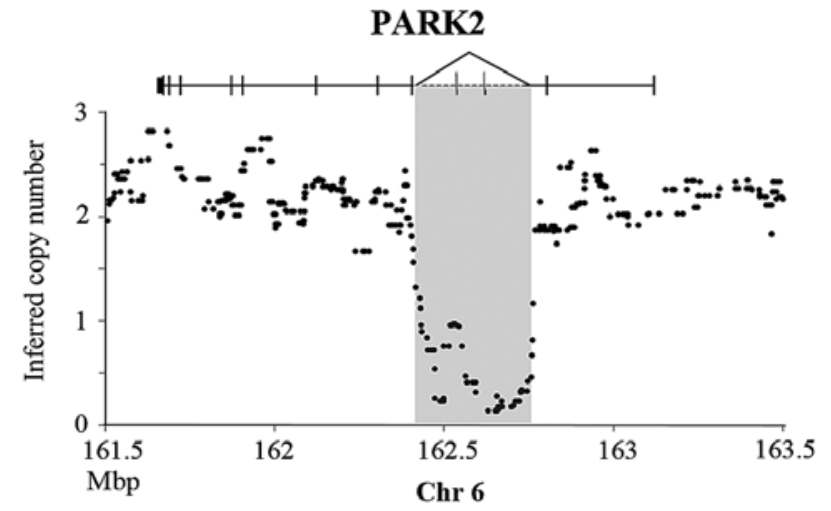

Figure 1. Refinement of the homozygous deletion breakpoints in PLC/PRF/5 by copy number variation. The copy number variation in the $P A R K 2$ region was calculated using the SNP probe intensity from the 500K SNP arrays. SNP, single nucleotide polymorphism.

culture medium in $60-\mathrm{mm}$ dishes. All of the experiments were conducted in triplicate. The dishes were incubated at $37^{\circ} \mathrm{C}$ in a $5 \% \mathrm{CO}_{2}$ incubator for 3 weeks, and the medium was changed every 3 days. The colonies were visualized with $1 \%$ crystal violet (Sigma-Aldrich) staining and photographed under light microscopy.

\section{Results}

To explore for fusion genes in cancer cells, we established stringent criteria for defining HD and amplicon breakpoints. First, the copy numbers for HDs and amplicon breakpoints were higher or lower than its neighboring probe for a copy number of at least 1.5 , respectively. Then, the breakpoints were located on a gene region. Overall, 80 breakpoints were identified and located in 39 genes (Table I). In total, 27 out of the 39 genes were identified to fuse with other genes by RNA-sequencing in cancer patients from the The Cancer Genome Atlas (TCGA) and COMIC fusion gene databases. These results suggest that most of the breakpoints in cancer are localized in common fragile regions. For example, Wang et al reported qPCR and fluorescence in situ hybridization (FISH) assay results showing that PARK2 lost exon 3 and 4 by the intra-chromosome rearrangement in the PLC/PRF/5 cell line (10). Our data not only confirmed the homozygous deletion in PLC/PRF/5, but also refined the breakpoints in PARK2 intron $2(162.47 \mathrm{Mb})$ and 4 (162.75 Mb) (Fig. 1).

In the present study, we focused on fusion genes produced by intra-chromosome rearrangement while inter-chromosome rearrangement results in complex products with difficult to predict fusion targets were not addressed. First, we selected the aberrant region where both the 5'- and 3'-end breakpoints were found and designed primers to check for potential fusion status using genomic PCR. The mean distance in a $500 \mathrm{~K}$ array is $\sim 5.8 \mathrm{~kb}$. Therefore, the breakpoints were detectable by genomic PCR. Finally, a potential fusion gene region was observed on chromosome 3q26 in Hep3B cells. We hypothesized that the breakpoints were in PRKCI intron 7 and $F N D C 3 B$ intron 12 after calculating the probe intensity on $3 \mathrm{q} 26$ for the copy number variation (Fig. 2A). Then, we designed primers targeting the intron of one of the genes to pair with a 
Table I. Breakpoints observed in cancer cell lines.

\begin{tabular}{|c|c|c|c|c|c|}
\hline Cell line & Chr & 5' Breakpoint & Gene & 3' Breakpoint & Gene \\
\hline \multicolumn{6}{|l|}{ Amplicons } \\
\hline $\mathrm{PLC} / \mathrm{PRF} / 5$ & 1 & 76795861 & ST6GALNAC3 & 76881390 & \\
\hline Hep3B & 3 & 171206621 & PRKCI & 173503700 & FNDC3B \\
\hline SNU387 & 3 & 7408728 & GRM7 & 7774941 & GRM7 \\
\hline $\mathrm{PLC} / \mathrm{PRF} / 5$ & 5 & 53314739 & ARL15 & 53499505 & \\
\hline $\mathrm{PLC} / \mathrm{PRF} / 5$ & 5 & 138510925 & SIL1 & 138694271 & \\
\hline Tong & 7 & 99315362 & & 99842479 & ZCWPW1 \\
\hline Tong & 7 & 107532265 & LAMB4 & 107578077 & \\
\hline Tong & 7 & 111630666 & DOCK4 & 112036838 & \\
\hline Hep3B & 8 & 31790956 & NRG1 & 35099889 & \\
\hline HA22T & 9 & 292324 & DOCKS & 416740 & \\
\hline HA22T & 9 & 12689776 & TYRP1 & 13017530 & MPDZ \\
\hline HA22T & 10 & 12486578 & PRESER2 & 12533424 & UPF2 \\
\hline HA22T & 10 & 12626943 & & 12761513 & CAMK1D \\
\hline Huh7 & 11 & 65636930 & PACS1 & 65754474 & \\
\hline SNU387 & 11 & 65846608 & PACS1 & 68628370 & \\
\hline Mahlavu & 16 & 76800885 & WWOX & 76844083 & \\
\hline Нер3B & 17 & 41625323 & & 41708649 & LRRC37A \\
\hline SK-Hep-1 & 17 & 41635580 & & 41708649 & LRRC37A \\
\hline SNU449 & 17 & 19109505 & & 19417180 & SLC47A1 \\
\hline HA22T & 19 & 40464311 & & 41239370 & WDR62 \\
\hline Mahlavu & 19 & 48855794 & & 51127600 & NOVA2 \\
\hline PLC/PRF/5 & 19 & 21336857 & ZNF708 & 22286718 & \\
\hline Tong & 19 & 39827914 & & 39895926 & ZNF599 \\
\hline HA59T & 20 & 29841434 & TPX2 & 30208930 & TPX2 \\
\hline Tong & 22 & 19458206 & PI4KA & 19520756 & \\
\hline HepG2 & $\mathrm{X}$ & 10576540 & MID1 & 10685154 & MID1 \\
\hline Huh6 & $\mathrm{X}$ & 29019665 & IL1RAPL1 & 29186133 & IL1RAPL1 \\
\hline \multicolumn{6}{|c|}{ Homozygous deletions } \\
\hline HA22T & 2 & 141590880 & LRP1B & 141738262 & LRP1B \\
\hline HA59T & 3 & 60520403 & FHIT & 60690206 & FHIT \\
\hline Нер3B & 3 & 53543957 & CACNA1D & 53646459 & CACNA1D \\
\hline Tong & 3 & 117643669 & LSAMP & 117718870 & LSAMP \\
\hline SNU398 & 4 & 93918392 & GRID2 & 94053969 & GRID2 \\
\hline PLC/PRF/5 & 6 & 162593039 & PARK2 & 162745092 & PARK2 \\
\hline HepG2 & 7 & 69045030 & AUTS2 & 69240841 & AUTS2 \\
\hline HepG2 & 7 & 78115615 & MAGI2 & 78249076 & MAGI2 \\
\hline HepG2 & 7 & 78581862 & MAGI2 & 78682833 & MAGI2 \\
\hline Mahlavu & 7 & 78153860 & MAGI2 & 78230785 & MAGI2 \\
\hline Tong & 8 & 3939796 & CSMD1 & 3947332 & CSMD1 \\
\hline Mahlavu & 9 & 9125223 & PTPRD & 9455190 & PTPRD \\
\hline SK-Hep-1 & 13 & 18983305 & & 20439027 & LATS2 \\
\hline
\end{tabular}

${ }^{a}$ Based on hg 18 human genome assembly. ${ }^{\mathrm{b}} \mathrm{Genes}$ which have been reported as fusion genes in TCGA or COSMIC databases are shown in bold. TCGA, The Cancer Genome Atlas; COSMIC, Catalogue of Somatic Mutations in Cancer.

primer for the exon of the other. Genomic PCR resulted in a 1.3-kb DNA fragment using the forward primer in $F N D C 3 B$ intron 12 and the reverse primer for PRKCI exon 8 (Fig. 2B).
The sequencing results suggested that the breakpoints were located at $169.95 \mathrm{Mb}(P R K C I)$ and $171.99 \mathrm{Mb}(F N D C 3 B)$, respectively. According to the orientation and localization 


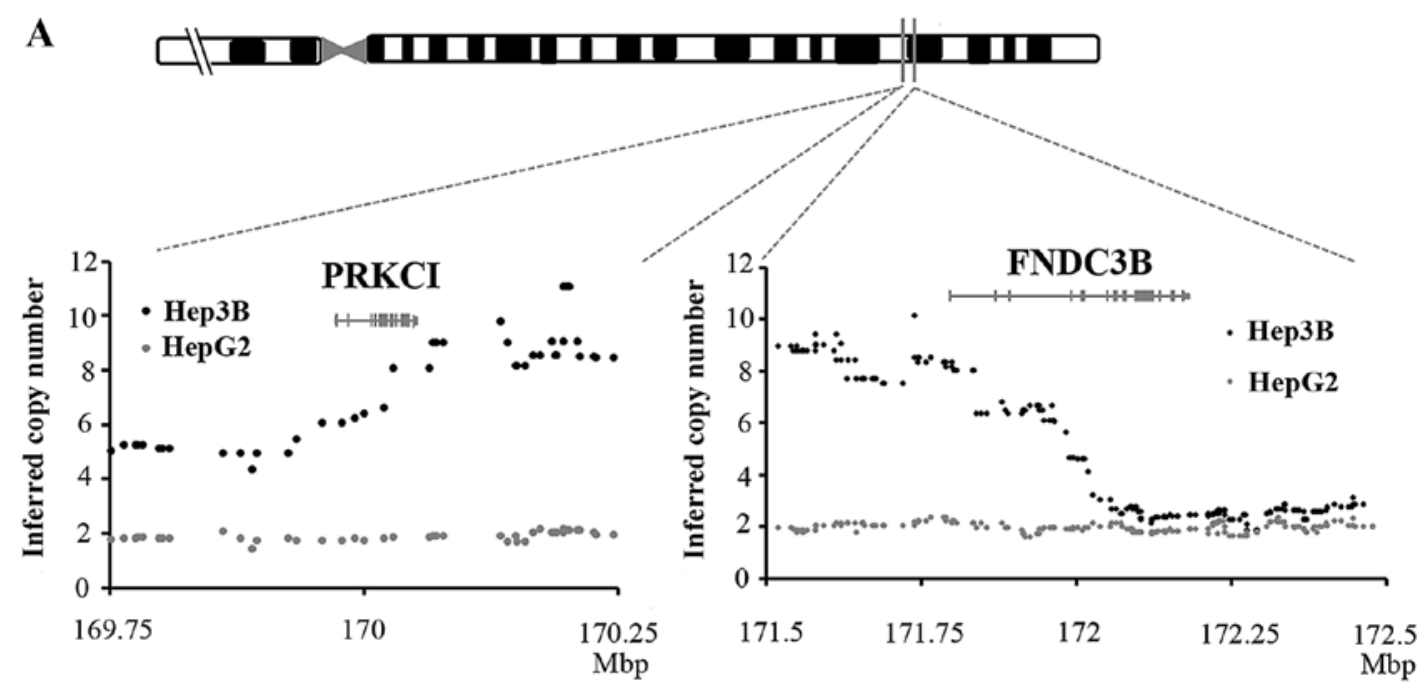

B

FNDC3B
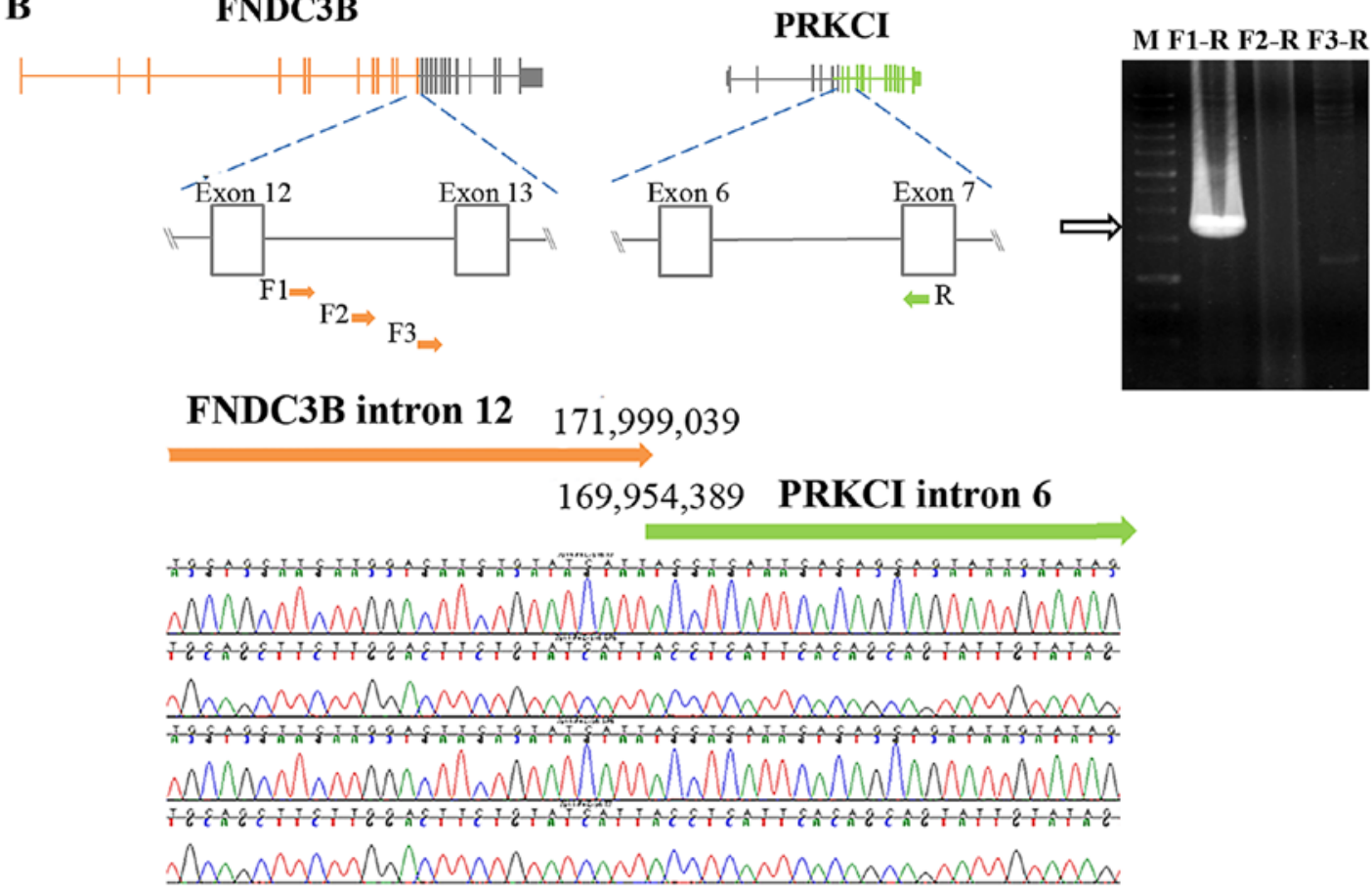

Figure 2. Copy number variation at 3q26 and sequence analyses of the chromosome breakpoints. (A) Copy number variation in the regions of FNDC3B and PRKCI were calculated using probe intensity from $500 \mathrm{~K}$ SNP arrays. (B) Three forward primers targeting $F N D C 3 B$ intron 12 and one reverse primer targeting PRKCI exon 7 were designed for genomic PCR. Then, the PCR product was sequenced to identify the chromosome breakpoints. FNDC3B, fibronectin type III domain containing 3B; PRKCI, protein kinase C iota; SNP, single nucleotide polymorphism.

of $P R K C I$ and $F N D C 3 B$ in chromosome 3 and our findings, we hypothesized that $F N D C 3 B-P R K C I$ results from a $2-\mathrm{Mb}$ segment duplication.

To establish our FNDC3B-PRKCI fusion model, we performed 3'-RACE PCR for $F N D C 3 B$ transcripts and detected a PCR product smaller than normal $F N D C 3 B$. Sequencing analyses revealed that the small transcripts were created by an in-frame fusion of $F N D C 3 B$ exons 1-12 with PRKCI exons 7-18 as predicted by our genomic DNA-sequencing results (Fig. 3A and B). Then, we performed western blotting using an antibody that specifically recognizes the FNDC3B $\mathrm{N}$-terminal region. FNDC3B expression in Hep3B cells was not different from other cell lines but an additional overexpressed band was observed at $\sim 90 \mathrm{kDa}$ (Fig. 3C).
As $F N D C 3 B-P R K C I$ was produced by in-frame fusion, the chimeric protein contained two complete fibronectin type III domains from $F N D C 3 B$ and the serine-threonine kinase domain from PRKCI (Fig. 3D). Both FNDC3B- and PRKCI-specific antibodies recognized the FNDC3B-PRKCI protein in Hep3B cells (Fig. 3C and E, left). A polyclonal antibody that recognizes the peptide across the FNDC3B-PRKCI fusion point was produced in rabbits to specifically detect the chimeric protein. Western blotting revealed that the antibody specifically detected the FNDC3B-PRKCI protein in Hep3B cells (Fig. 3E, right).

Huh6 cells were transfected with the fusion gene cloned into an expression vector to explore the oncogenic properties of FNDC3B-PRKCI. The 293T cell line was selected to express 
A

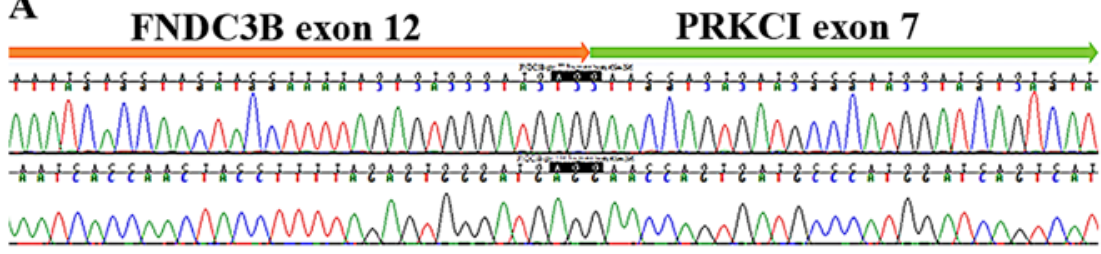

B

B FNDC3B

PRKCI

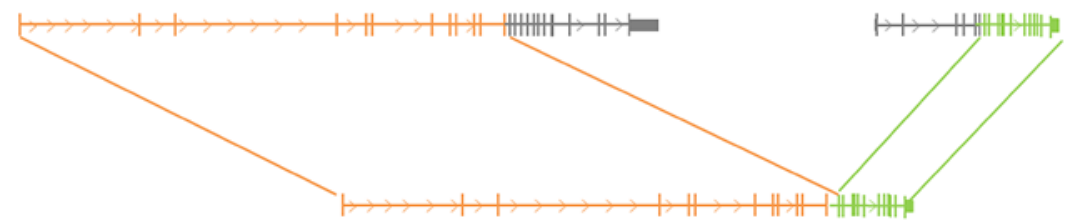

FNDC3B-PRKCI

C

Hep3B HepG3 Huh6 Huh7 SK-Hep-1

FNDC3B

$\beta$-actin

D

FNDC3B

PRKCI

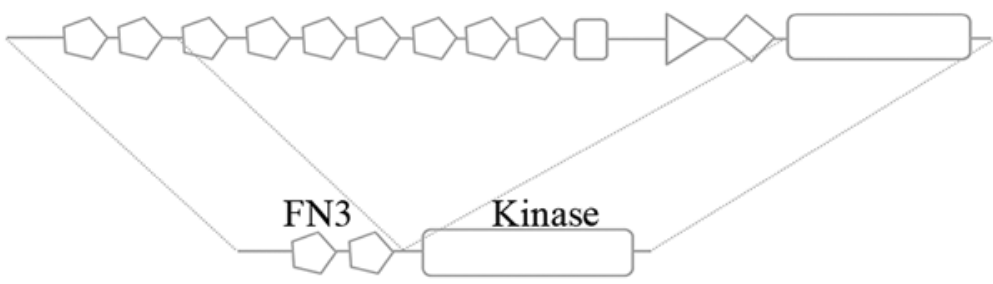

FNDC3B-PRKCI

E

Hep3B HepG2 Huh7

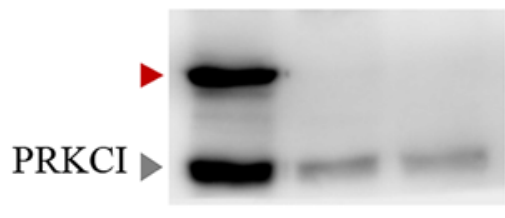

Tubulin

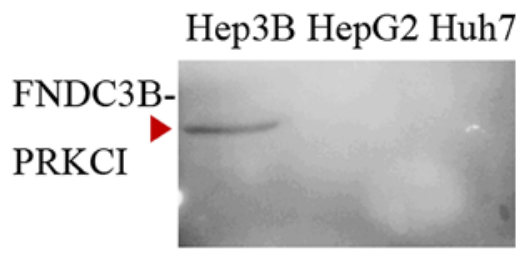

Tubulin

Figure 3. FNDC3B-PRKCI cDNA and protein structure. (A) A chimeric transcript was obtained by 3'-RACE PCR. The sequencing results indicated that it was a fusion product of FNDC3B and PRKCI. (B) The chimeric transcript was a fusion product of $F N D C 3 B$ and $P R K C I$ bases on the cDNA-sequencing result. (C) Western blotting of FNDC3B in the HCC cell lines. Gray triangle indicates the normal form ( 130 kDa) of FNDC3B and the red triangle indicates the novel fusion form $(\sim 100 \mathrm{kDa})$. (D) FNDC3B-PRKCI contains two fibronectin type III domains from FNDC3B and a serine-threonine kinase domain from PRKCI. FN3, fibronectin type III domains: TM, transmembrane domain $(\square)$ and PB1, Phox and Bem1p domain ( $\triangleright$ ); kinase, serine-threonine kinase domain: C1, protein kinase C conserved region 1 domain $(\diamond)$. (E) Western blotting revealed that FNDC3B-PRKCI was detectable by PRKCI antibody and our FNDC3B-PRKCI-specific antibody. Gray triangle indicates the normal form ( $70 \mathrm{kDa})$ of PRKCI, and red triangle indicates the novel fusion form ( 100 kDa). FNDC3B, fibronectin type III domain containing 3B; PRKCI, protein kinase C iota; 3'-RACE,3'-rapid amplification of cDNA ends.

the fusion protein since it was deficient in FNDC3B (data not shown). FNDC3B-PRKCI did not exert any significant effect on either Huh6 or 293T cells in regards to cell growth (Fig. 4A). However, overexpression of FNDC3B-PRKCI significantly enhanced cell migration (Fig. 4B) and increased the number of colonies as revealed in the anchorage-independent growth assay (Fig. 4C). These results indicate that FNDC $3 B-P R K C I$ is a potential oncogene in $\mathrm{HCC}$.

\section{Discussion}

After predicting chromosome breakpoints and performing genomic PCR and sequencing analyses, we suggest a potential chromosome intra-rearrangement model at chromosome $3 q 26$ in Hep3B cells. A chimeric transcript was observed by 3'-RACE analysis and the FNDC3B-PRKCI in-frame fusion protein was detected by western blotting. Finally, results of 
A

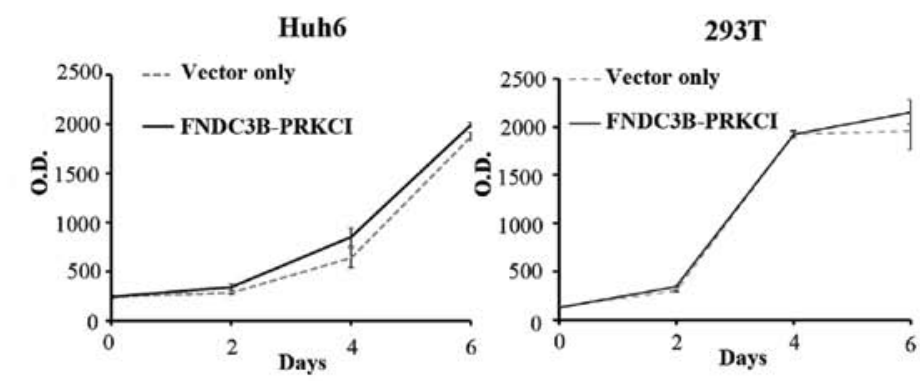

B
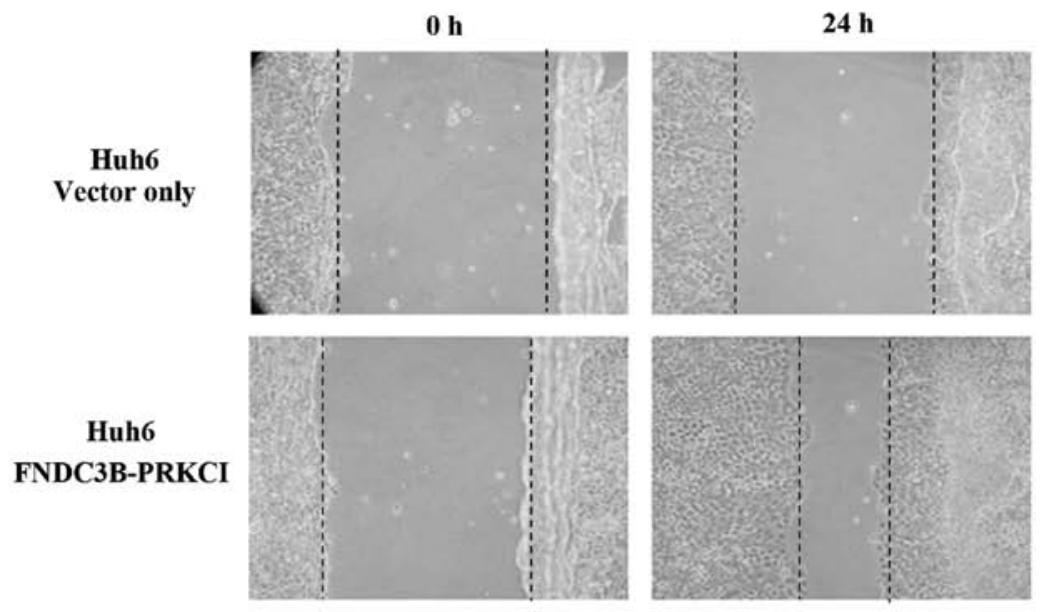

C

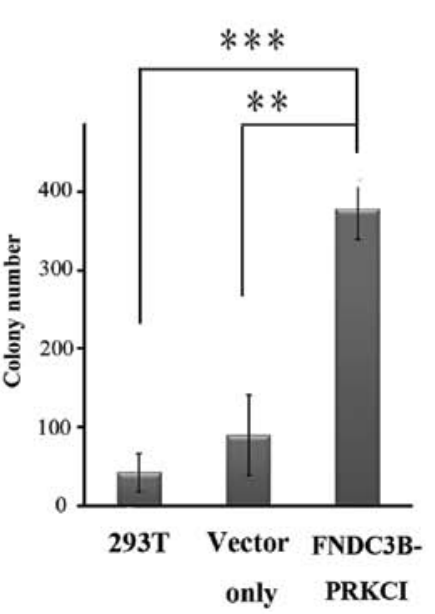

Figure 4. FNDC3B-PRKCI exhibits oncogenic potential. (A) A cell growth assay was performed using Huh6 and 293T cells transfected with $F N D C 3 B-P R K C I$ (B) A wound-healing assay was performed using Huh6 cells transfected with FNDC3B-PRKCI. Migration ability was determined by calculating the area of cell coverage after $24 \mathrm{~h}$ of incubation. (C) Colony formation was observed in anchorage-independent growth assays of 293T cells transfected with FNDC3B-PRKCI; ${ }^{* *} \mathrm{P}<0.001$ and ${ }^{* * * *} \mathrm{P}<0.0001$, denote a significant difference. FNDC3B, fibronectin type III domain containing 3B; PRKCI, protein kinase C iota.

cell migration and colony formation assays suggested that FNDC3B-PRKCI is a potential oncogene.

Fusion genes created by joining two functional fragments from different genes can provide a handy genetic window for dissecting novel pathways involved in tumorigenesis. Moreover, they can be used as diagnostic markers for cancer and targeted therapy. However, it is difficult to explore fusion genes and chromosome breakpoints in solid tumors. One major reason is that solid tumors are commonly contaminated with normal or pre-malignant tissues (3). For purity and convenience, searching for fusion candidates in tumor cell lines and confirming their presence in tumor samples using high-throughput screening is a better solution (3). For example, recurrence breakpoints for NRG1 were first found in breast and prostate cancer cell lines (11). Then, they were discovered in breast cancer samples at a follow-up screening using tissue assays and paraffin-embedded tissue samples $(12,13)$. Gene fusion is caused by complex and small chromosomal rearrangements such as duplications, deletions and insertions (3). These small rearrangements cannot be detected by traditional low resolution cytogenetic methods, but are detectable by new high-throughput sequencing or array platforms. Therefore, several new fusion genes in solid tumors have recently been reported. For example, the first fusion gene in HCC, ABCB11-LRP2, was detected by whole-genome sequencing (14). We found genomic breakpoints by applying 
high-resolution arrays and predicted a $2-\mathrm{Mb}$ segment duplication model in chromosome 3q26. Notably, the same genomic breakpoints occurred in at least $3 \mathrm{HCC}$ patients by analysis data from TCGA HCC patients (data not shown).

$F N D C 3 B$ is also named factor for adipocyte differentiation 104 (FAD104) for it has been identified as a regulator of differentiation in adipocytes and osteoblasts $(15,16)$. This gene is commonly overexpressed in many types of cancer (17), but its biological functions remain largely unknown. FNDC3B is mainly composed of nine FNIII domains and one transmembrane domain. In terms of function, most of the FNIII domains are commonly involved in cell adhesion and growth signaling $(17,18)$. One study suggested that $F N D C 3 B$ is involved in epithelial-mesenchymal transition (EMT) (19), a cellular process in which epithelial cells lose their polarized organization and acquire mesenchymal characteristics. EMT is a critical process for the conversion of early-stage tumors into invasive malignancies $(20,21)$. In our experiments, FNDC3B-PRKCI enhanced cell migration and colony formation (Fig. 4B and C) suggesting that the first two FNIII domains in FNDC3B are important for the EMT process.

Protein kinase $\mathrm{C}(\mathrm{PKC})$ is a family of serine/threonine protein kinases. PRKCI belongs to an atypical PKC subgroup in which catalytic activity is not dependent on diacylglycerol, calcium or phosphatidylserine (22). Accumulating evidence indicates that $P R K C I$ is an oncogene and a prognostic marker in many human types of cancer, such as lung, ovarian, prostate, gastric, breast and liver cancer (23-28). PRKCI is required for Ras-mediated transformation and it participates in multiple aspects of the transformed phenotype including growth, invasion and survival (29,30). For example, PRKCI-mediated phosphorylation of $\mathrm{I} \kappa \mathrm{K}$ leads to activation of the canonical $\mathrm{NF}-\kappa \mathrm{B}$ pathway and cell survival in prostate cancer (31). The presence of a complete serine-threonine kinase domain in FNDC3B-PRKCI suggests that the chimeric protein can transduce signals via phosphorylation. However, further kinase activity and protein-protein interaction studies are required to understand the detailed molecular mechanism of FNDC3B-PRKCI in tumorigenesis.

In the present study, we combined genomic and proteomic approaches to identify a novel fusion gene at the DNA, RNA and protein levels. Functional-assay results suggest that $F N D C 3 B-P R K C I$ is a potential oncogene. In addition, a specific antibody for $F N D C 3 B-P R K C I$ was developed, which could be beneficial for further patient screening.

\section{Acknowledgements}

The present study was funded by grants from the Ministry of Science and Technology, Taiwan (NSC 101-2320-B-010066-MY3, MOST 104-2314-B-532-005- and MOST 105-2319-B-010-001), and the National Yang-Ming University, Taiwan (Ministry of Education, Aim for the Top University Plan). The authors acknowledge the technical services provided by the High-Throughput Genome Analysis Core Facility of VYM Genome Research Center (VYMGC), National Yang-Ming University. The Core Facility was supported by the National Core Facility Program for Biotechnology (NCFPB), Ministry of Science and Technology.

\section{References}

1. Kaye FJ: Mutation-associated fusion cancer genes in solid tumors. Mol Cancer Ther 8: 1399-1408, 2009.

2. Prensner JR and Chinnaiyan AM: Oncogenic gene fusions in epithelial carcinomas. Curr Opin Genet Dev 19: 82-91, 2009.

3. Edwards PA: Fusion genes and chromosome translocations in the common epithelial cancers. J Pathol 220: 244-254, 2010.

4. Licht JD: Acute promyelocytic leukemia - weapons of mass differentiation. N Engl J Med 360: 928-930, 2009.

5. Nambiar M, Kari V and Raghavan SC: Chromosomal translocations in cancer. Biochim Biophys Acta 1786: 139-152, 2008.

6. Heim S and Mitelman F: Molecular screening for new fusion genes in cancer. Nat Genet 40: 685-686, 2008.

7. Howarth KD, Blood KA, Ng BL, Beavis JC, Chua Y, Cooke SL, Raby S, Ichimura K, Collins VP, Carter NP, et al: Array painting reveals a high frequency of balanced translocations in breast cancer cell lines that break in cancer-relevant genes. Oncogene 27: 3345-3359, 2008

8. Maher CA, Kumar-Sinha C, Cao X, Kalyana-Sundaram S, Han B, Jing X, Sam L, Barrette T, Palanisamy N and Chinnaiyan AM: Transcriptome sequencing to detect gene fusions in cancer. Nature 458: 97-101, 2009.

9. Chen CF, Hsu EC, Lin KT, Tu PH, Chang HW, Lin CH, Chen YJ, Gu DL, Lin CH, Wu JY, et al: Overlapping high-resolution copy number alterations in cancer genomes identified putative cancer genes in hepatocellular carcinoma. Hepatology 52: 1690-1701, 2010.

10. Wang F, Denison S, Lai JP, Philips LA, Montoya D, Kock N, Schüle B, Klein C, Shridhar V, Roberts LR, et al: Parkin gene alterations in hepatocellular carcinoma. Genes Chromosomes Cancer 40: 85-96, 2004.

11. Adélaïde J, Huang HE, Murati A, Alsop AE, Orsetti B, Mozziconacci MJ, Popovici C, Ginestier C, Letessier A, Basset C, et al: A recurrent chromosome translocation breakpoint in breast and pancreatic cancer cell lines targets the neuregulin/NRG1 gene. Genes Chromosomes Cancer 37: 333-345, 2003.

12. Huang HE, Chin SF, Ginestier C, Bardou VJ, Adélaïde J, Iyer NG, Garcia MJ, Pole JC, Callagy GM, Hewitt SM, et al: A recurrent chromosome breakpoint in breast cancer at the NRG1/neuregulin 1/heregulin gene. Cancer Res 64: 6840-6844, 2004.

13. Prentice LM, Shadeo A, Lestou VS, Miller MA, deLeeuw RJ, Makretsov N, Turbin D, Brown LA, Macpherson N, Yorida E, et al: NRG1 gene rearrangements in clinical breast cancer: Identification of an adjacent novel amplicon associated with poor prognosis. Oncogene 24: 7281-7289, 2005.

14. Fernandez-Banet J, Lee NP, Chan KT, Gao H, Liu X, Sung WK, Tan W, Fan ST, Poon RT, Li S, et al: Decoding complex patterns of genomic rearrangement in hepatocellular carcinoma. Genomics 103: 189-203, 2014.

15. Tominaga $\mathrm{K}$, Kondo $\mathrm{C}$, Johmura $\mathrm{Y}$, Nishizuka $\mathrm{M}$ and Imagawa $\mathrm{M}$ : The novel gene fad104, containing a fibronectin type III domain, has a significant role in adipogenesis. FEBS Lett 577: 49-54, 2004.

16. Kishimoto K, Kato A, Osada S, Nishizuka $M$ and Imagawa $M$ : Fad104, a positive regulator of adipogenesis, negatively regulates osteoblast differentiation. Biochem Biophys Res Commun 397: 187-191, 2010.

17. Lin F, Ren XD, Pan Z, Macri L, Zong WX, Tonnesen MG, Rafailovich M, Bar-Sagi D and Clark RA: Fibronectin growth factor-binding domains are required for fibroblast survival. J Invest Dermatol 131: 84-98, 2011.

18. Obara M, Sakuma T and Fujikawa K: The third type III module of human fibronectin mediates cell adhesion and migration. $\mathrm{J}$ Biochem 147: 327-335, 2010.

19. Cai C, Rajaram M, Zhou X, Liu Q, Marchica J, Li J and Powers RS: Activation of multiple cancer pathways and tumor maintenance function of the $3 \mathrm{q}$ amplified oncogene $F N D C 3 B$. Cell Cycle 11: 1773-1781, 2012.

20. Yang $\mathbf{J}$ and Weinberg RA: Epithelial-mesenchymal transition: At the crossroads of development and tumor metastasis. Dev Cell 14: 818-829, 2008.

21. Pinzani M: Epithelial-mesenchymal transition in chronic liver disease: Fibrogenesis or escape from death? J Hepatol 55: 459-465, 2011.

22. Steinberg SF: Structural basis of protein kinase C isoform function. Physiol Rev 88: 1341-1378, 2008. 
23. Win HY and Acevedo-Duncan M: Role of protein kinase C-iota in transformed non-malignant RWPE-1 cells and androgen-independent prostate carcinoma DU-145 cells. Cell Prolif 42: 182-194, 2009.

24. Takagawa R, Akimoto K, Ichikawa Y, Akiyama H, Kojima Y Ishiguro H, Inayama Y, Aoki I, Kunisaki C, Endo I, et al: High expression of atypical protein kinase $\mathrm{C}$ lambda/iota in gastric cancer as a prognostic factor for recurrence. Ann Surg Oncol 17: $81-88,2010$.

25. Kikuchi K, Soundararajan A, Zarzabal LA, Weems CR, Nelon LD, Hampton ST, Michalek JE, Rubin BP, Fields AP and Keller C: Protein kinase C iota as a therapeutic target in alveolar rhabdomyosarcoma. Oncogene 32: 286-295, 2013.

26. Justilien V, Walsh MP, Ali SA, Thompson EA, Murray NR and Fields AP: The PRKCI and SOX2 oncogenes are coamplified and cooperate to activate Hedgehog signaling in lung squamous cell carcinoma. Cancer Cell 25: 139-151, 2014.
27. Haverty PM, Hon LS, Kaminker JS, Chant J and Zhang Z: High-resolution analysis of copy number alterations and associated expression changes in ovarian tumors. BMC Med Genomics 2: 21, 2009.

28. Du GS, Wang JM, Lu JX, Li Q, Ma CQ, Du JT and Zou SQ: Expression of P-aPKC-iota, E-cadherin, and beta-catenin related to invasion and metastasis in hepatocellular carcinoma. Ann Surg Oncol 16: 1578-1586, 2009.

29. Fields AP and Regala RP: Protein kinase C iota: Human oncogene, prognostic marker and therapeutic target. Pharmacol Res 55: 487-497, 2007.

30. Murray NR, Kalari KR and Fields AP: Protein kinase $C_{t}$ expression and oncogenic signaling mechanisms in cancer. J Cell Physiol 226: 879-887, 2011.

31. Win HY and Acevedo-Duncan M: Atypical protein kinase C phosphorylates IKKalphabeta in transformed non-malignant and malignant prostate cell survival. Cancer Lett 270: 302-311, 2008. 